\title{
Art. 22a KVG: Fluch oder Segen?
}

Esther Kraft

Korrespondenz: Esther Kraft FMH / Abteilung DDQ Elfenstrasse 18 CH-3000 Bern 15 Tel. 0313591111 Fax 0313591112 esther.kraft@fmh.ch
Seit dem 1. Januar 2009 gilt im Krankenversicherungsgesetz (KVG) ein neuer Artikel: Art. 22a KVG sowie der dazugehörige Artikel in der Verordnung über die Krankenversicherung (KVV): Art. $31 \mathrm{KVV}$.

Gemäss Art. 22a KVG sind die Leistungserbringer verpflichtet, den zuständigen Bundesämtern Daten bekanntzugeben, die benötigt werden, um die Wirtschaftlichkeit und Qualität der Leistungen zu überwachen. Es sind unter anderem Angaben zur Art der Tätigkeit, Patientenstruktur, zu Art, Umfang und Kosten der erbrachten Leistungen sowie Angaben zu Qualitätsindikatoren.

Es sollen Daten oder Kennzahlen zu folgenden Themen kommuniziert werden:

- Leistungsangebot der Leistungserbringer

- Diplome und Weiterbildungstitel der Leistungserbringer

- Medizinische Qualitätsindikatoren

- Umfang und Art der erbrachten Leistungen

- Kostenentwicklung

Für die Erhebung und Sammlung der Daten ist das Bundesamt für Statistik (BFS) verantwortlich. Gemäss Art. $31 \mathrm{KVV}$ kann das Bundesamt für Gesundheit (BAG) die Zahlen veröffentlichen. Die Details der Umsetzung des Artikels sind bis heute unklar. Ziel ist es, erste Zahlen und Indikatoren im Jahr 2013 zu publizieren.

\section{Das Wichtigste in Kürze}

- Art. 22a KVG ist seit 1.1.2009 in Kraft;

- Leistungserbringer sind verpflichtet, verschiedene Daten dem Bund bekanntzugeben;

- FMH will Aufwand für die Ärzteschaft minimal halten;

- FMH koordiniert innerhalb der Ärzteschaft;

- FMH arbeitet bei der Umsetzung des Artikels mit.

Die Transparenz der Datengrundlagen, das Zusammenführen der Daten und die allfällige anschliessende Interpretation der Daten sind wichtige Elemente der Publikation. Bei der Erfüllung des neuen KVG-Artikels müssen die verschiedenen Datenlieferanten miteinbezogen werden und Anpassungen in der Methodik, Auswertungen usw. sind möglich.

Deshalb hat die FMH beschlossen, innerhalb der Ärzteschaft die Federführung bei der Umsetzung des Artikels 22a KVG zu übernehmen und betraute die Abteilung Daten, Demographie und Qualität (DDQ) mit dieser Aufgabe. Bereits im Mai 2009 hat die FMH zusammen mit Vertretern von NewIndex, Trustcenter und KKA/ROKO [1] eine Arbeitsgruppe gegründet. Diese erstellte einen Überblick über die in der Ärzteschaft vorhandenen Daten und bewertete diese bzgl.

\section{Transparenz und Vergleichbarkeit ärztlicher Leistungen sind zu begrüssen - aber nur, wenn sie auf zuverlässigen und wissenschaftlich fundierten Zahlen beruhen}

Vor allem die ambulant tätigen Ärztinnen und Ärzte sind von diesem Artikel direkt betroffen. Für die FMH ist es daher ein grosses Anliegen, dass bei der Umsetzung für die Ärzteschaft kein unnötiger bürokratischer Aufwand entsteht. In den verschiedenen Organisationen und Institutionen der Ärzteschaft sind bereits viele Daten und Angaben vorhanden, die zur Zeit aber noch wenig koordiniert sind. Für die Erfüllung dieses Artikels ist es für die FMH zentral, dass bereits vorhandene Daten verwendet werden und nicht zwingend neue Datenerhebungen erfolgen. Die vorhandenen Routinedaten dürfen aber nicht einfach 1:1 für die Erfüllung des Artikels angewendet werden. ihrer Qualität, ihrer Verfügbarkeit und ihrer Möglichkeiten zur Erfüllung des Artikels 22a KVG. Da das BFS für die Erhebung zuständig ist, hat die FMH bereits früh mit den zuständigen Stellen Kontakt aufgenommen und ihre Mitarbeit offeriert. Diese ist bei der Umsetzung des Artikels für die Ärzteschaft zwingend nötig, denn nur die FMH kann den grossen Koordinationsaufwand zwischen den verschiedenen Organisationen bewältigen und es ist wichtig, dass die Ärzteschaft in dieser Thematik mit einer Stimme spricht. Durch die Mitarbeit der FMH kann gewährleistet werden, dass der administrative Aufwand für die Ärzteschaft und für deren Organisationen minimal bleibt. 


\section{Art. 22a KVG Daten der Leistungserbringer [3]}

Die Leistungserbringer sind verpflichtet, den zuständigen Bundesbehörden die Daten bekanntzugeben, die benötigt werden, um die Anwendung der Bestimmungen dieses Gesetzes über die Wirtschaftlichkeit und Qualität der Leistungen zu überwachen. Namentlich sind folgende Angaben zu machen:

a. Art der Tätigkeit, Einrichtung und Ausstattung, Rechtsform;

b. Anzahl und Struktur der Beschäftigten und der Ausbildungsplätze;

c. Anzahl und Struktur der Patientinnen und Patienten in anonymisierter Form;

d. Art, Umfang und Kosten der erbrachten Leistungen;

e. Aufwand, Ertrag und finanzielles Betriebsergebnis;

f. medizinische Qualitätsindikatoren.

Die befragten natürlichen und juristischen Personen sind zur Auskunft verpflichtet. Die Angaben sind kostenlos zur Verfügung zu stellen.

Die Angaben werden vom Bundesamt für Statistik erhoben. Es stellt die Angaben nach Absatz 1 zur Durchführung dieses Gesetzes dem Bundesamt für Gesundheit, dem Eidgenössischen Preisüberwacher, dem Bundesamt für Justiz, den Kantonen und Versicherern sowie den in Artikel 84a aufgeführten Organen je Leistungserbringer zur Verfügung. Die Daten werden veröffentlicht.

4 Der Bundesrat erlässt nähere Vorschriften zur Erhebung, Bearbeitung, Weitergabe und Veröffentlichung der Daten unter Wahrung des Verhältnismässigkeitsprinzips.

\section{Art. 23 KVG Statistiken [3]}

Das Bundesamt für Statistik erarbeitet die notwendigen statistischen Grundlagen zur Beurteilung von Funktions- und Wirkungsweise dieses Gesetzes. Es erhebt zu diesem Zweck bei den Versicherern, den Leistungserbringern und der Bevölkerung die notwendigen Daten.

2 Die befragten natürlichen und juristischen Personen sind zur Auskunft verpflichtet. Die Informationen sind kostenlos zur Verfügung zu stellen.

3 Das Bearbeiten von Daten zu statistischen Zwecken erfolgt nach dem Bundesstatistikgesetz vom 9. Oktober 1992.

\section{Art. 31 KVV Veröffentlichung der Daten der Leistungserbringer [4]}

Das BAG veröffentlicht die Ergebnisse der vom Bundesamt für Statistik gestützt auf Artikel 22a des Gesetzes und durch das BAG nach Artikel 51 des Bundesgesetzes vom 26. Juni 2006 über die universitären Medizinalberufe erhobenen Daten so, dass namentlich folgende Angaben oder Kennzahlen der sozialen Krankenversicherung nach

Leistungserbringer oder nach Kategorien von Leistungserbringern ersichtlich sind:

a. Leistungsangebot der Leistungserbringer;

b. Diplome und Weiterbildungstitel der Leistungserbringer;

c. medizinische Qualitätsindikatoren;

d. Umfang und Art der erbrachten Leistungen;

e. Kostenentwicklung.
Es ist nicht im Sinne der Ärzteschaft, dass infolge der Einführung dieses Artikel unkoordinierte und unausgereifte Zahlen, wie z.B. die Mortalitätsstatistik des Bundes, veröffentlicht werden. Eine Erarbeitung von sinnvollen und wissenschaftlichen Indikatoren und Methoden in Zusammenarbeit mit den medizinischen Fachgesellschaften und den anderen Akteuren des Gesundheitswesens ist unabdingbar.

Nur mit einem ausgereiften und überlegten Vorgehen bei der Umsetzung des Art. 22a KVG können die Kennzahlen/Indikatoren zu einem Segen für das Gesundheitssystem Schweiz führen - dank verbesserter Transparenz und fundierten Grundlagen. Nimmt man hingegen eine Handvoll Routinedaten, vermischt diese beliebig miteinander und würzt sie mit einer Prise Ignoranz, kann das Vorhaben sehr schnell zum Fluch für alle werden [2].

Die Abteilung DDQ der FMH (ddq@fmh.ch) steht allen Ärztinnen und Ärzten, den kantonalen und fachspezifischen Ärztegesellschaften, den Basisorganisationen usw. für Fragen und Informationen zur Verfügung und stellt gerne bestehende Unterlagen zu.

\section{Literatur}

1 KKA: Konferenz der kantonalen Ärztegesellschaften / ROKO: Rollende Kostenstudie.

2 Herren D. Spiel mir das Lied der Mortalitätsstatitik ... Schweiz Ärztezeitung. 2009;90(17):649.

3 Bundesgesetz über die Krankenversicherung (KVG) www.admin.ch/ch/d/sr/832_10/vom 10.9.2010.

4 Verordnung über die Krankenversicherung (KVV), www.admin.ch/ch/d/sr/832 102/ vom 10.9.2010. 